\title{
Estrategias y materiales didácticos digitales para estudiantes en educación infantil con diversidad de aprendizaje
}

\author{
Digital teaching strategies and materials for student learning in early childhood education with \\ learning diversity
}

Estratégias e materiais digitais de ensino para estudantes na educação infantil com diversidade de aprendizage

Recibido: 11/01/2021 | Revisado: 11/01/2021 |Acepto: 13/01/2021 | Publicado: 17/01/2021

\author{
Karina Maribel Castillo Pinos \\ ORCID: https://orcid.org/0000-0002-1154-1297 \\ Universidad Nacional de Educación, Ecuador \\ E-mail: karina.castillo@unae.edu.ec \\ Derling Jose Mendoza Velazco \\ ORCID: https://orcid.org/0000-0001-8275-3687 \\ Universidad UTE, Ecuador \\ Universidad Nacional de Educación, Ecuador \\ E-mail: derling.mendoza@ute.edu.ec
}

\begin{abstract}
Resumen
El estudio llevado a cabo tuvo como finalidad determinar la relación de las acciones estratégicas y materiales didácticos digitales implementados pos los facilitadores o docentes sobre el aprendizaje de los alumnos de 3 a 6 años con diversidad de aprendizaje en la ciudad de Azogues. En este caso, el enfoque del estudio fue cuantitativo y de tipo descriptivo correlacional de Pearson, mientras que el diseño aplicado fue de tipo no experimental transeccional. Se trabajó con una población compuesta de 12 alumnos con discapacidad intelectual, considerando la aplicación de la ficha de observación, para lo cual se diseñaron dos formatos de preguntas direccionadas a evaluar cada una de las variables estudiadas. Los resultados de la correlación de Pearson demostraron que las estrategias y materiales didácticos (EMD) están relacionas favorablemente en un orden de 0.639 con el aprendizaje en los niños con diversidad de aprendizaje (ADI); en cuyo caso se identificó que la correlación es significativa en el nivel 0,05 bilateral. No obstante, con base a la discusión realizada se determinó que las estrategias y materiales didácticos digitales empleados deben ser aplicadas de manera más eficiente para obtener mejores resultados a nivel de aprendizaje, principalmente en el caso de los estudiantes que presentan mayor grado de diversidad de aprendizaje, puesto que de acuerdo con la investigación muestran mayores dificultades de aprendizaje.
\end{abstract}

Palabras clave: Deficiencia cognitiva; Didáctica; Diversidad de aprendizaje; Educación integradora.

\begin{abstract}
The purpose of the study carried out was to determine the relationship of the strategic actions and digital teaching materials implemented by the facilitators or teachers on the learning of students aged 3 to 6 with intellectual disabilities in the city of Azogues. In this case, the study approach was quantitative and Pearson's correlational descriptive type, while the applied design was non-experimental, transectional. We worked with a population composed of 12 students with intellectual disabilities, considering the application of the observation sheet, for which two formats of questions were designed aimed at evaluating each of the variables studied. The results of the Pearson correlation demonstrated that teaching strategies and materials (DME) are favorably related in an order of 0.639 with learning in children with intellectual disabilities (ADI); in which case the correlation was found to be significant at the bilateral 0.05 level. However, based on the discussion carried out, it was determined that the digital teaching materials and strategies used should be applied more efficiently to obtain better results at the learning level, mainly in the case of students with a higher degree of intellectual disability since according to research they show greater learning difficulties.
\end{abstract}

Keywords: Cognitive impairment; Didactic; Diversity of learning; Inclusive education.

\section{Resumo}

O objectivo do estudo era determinar a relação entre as acções estratégicas e os materiais didácticos digitais implementados pelos facilitadores ou professores sobre a aprendizagem dos estudantes dos 3 aos 6 anos de idade com a diversidade de aprendizagem na cidade de Azogues. Neste caso, o foco do estudo foi quantitativo e do tipo descritivo correlacional de Pearson, enquanto que o desenho aplicado foi de um tipo transeccional não-experimental. 
Trabalhámos com uma população composta por 12 estudantes com deficiência intelectual, considerando a aplicação do cartão de observação, para o qual foram concebidos dois formatos de perguntas para avaliar cada uma das variáveis estudadas. Os resultados da correlação de Pearson mostraram que as estratégias e materiais didácticos (DMS) estão favoravelmente relacionados numa ordem de 0,639 à aprendizagem em crianças com diversidade de aprendizagem (LDR); neste caso, a correlação foi identificada como significativa ao nível bilateral de 0,05. Contudo, com base na discussão realizada, foi determinado que as estratégias e materiais de ensino digitais utilizados deveriam ser aplicados de forma mais eficiente para obter melhores resultados ao nível da aprendizagem, principalmente no caso dos estudantes que apresentam um maior grau de diversidade de aprendizagem, uma vez que, de acordo com a investigação, apresentam maiores dificuldades de aprendizagem.

Palavras-chave: Deficiência cognitiva; Didáctica; Diversidade da aprendizagem; Educação inclusiva.

\section{Introducción}

El aprendizaje inclusivo se propicia a partir del acatamiento de las políticas nacionales e internacionales dispuestas para garantizar la inclusión de los estudiantes con discapacidad al sistema educativo formal, en cumplimiento con los derechos humanos que determinan el derecho de todos los individuos de acceder a una educación de calidad, disfrutar de una vida digna en plenitud de condiciones (Bautista \& Clavijo, 2020).

La diversidad de aprendizaje se refiere a la infinita variedad de experiencias y atributos de la vida que un niño aporta a su aprendizaje formal en la escuela. Todos los estudiantes con diversas necesidades de aprendizaje tienen derecho a acceder a una educación completa y atractiva en la misma base que sus compañeros. Abdul \& Mohammed, (2019) reconocen que a veces las necesidades de aprendizaje académico, físico o social y emocional de un estudiante pueden ser muy diferentes de las de sus compañeros. Estas diferencias pueden estar relacionadas con, trasfondo cultural, estatus socioeconómico, necesidades de aprendizaje, habilidad excepcional, discapacidad física, cognitiva, social y emocional o sensorial.

Debido a las políticas vigentes, en diversas instituciones educativas se gestan programas en los que resulta especialmente importante el desarrollo de directrices para la inclusión de niños con discapacidades actualizando el contenido de la educación de acuerdo con las necesidades de la población de estudiantes con discapacidades, además del uso de materiales didácticos, especialmente por el potencial para mejorar el aprendizaje que poseen (Figueroa, Piñeres, \& Velásquez, 2017).

Estas decisiones políticas y los programas de desarrollo educativo basados en ellas reflejan la conciencia de la necesidad de abordar el problema de crear un entorno educativo inclusivo para los niños y la obligación de brindarles una educación de calidad desde la etapa de educación infantil y promover el aprendizaje permanente (Bautista \& Clavijo, 2020). Es necesario recalcar que la inclusión en todos los ámbitos de la sociedad es una tarea relevante en función de promover el bienestar de los niños con discapacidades. En este sentido, el uso de las tecnologías de la información y la comunicación (TIC's) pueden proporcionar un mayor acceso al conocimiento, a su vez pone a disposición una amplia gama de herramientas que facilitan el acompañamiento por parte del docente al estudiante con discapacidades, de modo que su uso puede mejorar la calidad de la educación (Molina, 2015).

Una definición de referencia de estilos de aprendizaje es comportamientos cognitivos, efectivos y psicosociales característicos que sirven como indicadores relativamente estables de cómo los alumnos perciben, interactúan y responden al entorno de aprendizaje. Romanelli, Bird, \& Ryan, (2009), consideran que los estilos de aprendizaje son un factor de éxito en la educación, la investigación educativa y, en muchos casos, la aplicación de la teoría del estilo de aprendizaje ha engendrado la mirada de métodos utilizados para categorizar los estilos de aprendizaje con estudiantes especiales. Actualmente no existe un método único comúnmente aceptado, pero alternativamente se están utilizando varias escalas y clasificaciones potenciales. La mayoría de estas escalas y clasificaciones son más similares que diferentes y se centran en las preferencias ambientales, las modalidades sensoriales, los tipos de personalidad y / o los estilos cognitivos.

Siguiendo el mismo orden de ideas, es fundamental considerar que para lograr una verdadera inclusión, resulta 
imperativo además de proporcionar todos los recursos necesarios para garantizar una educación de calidad; garantizar el financiamiento para la formación constante del docente y el acceso a herramientas y materiales didácticos digitales adecuados, a su vez es preciso fomentar la inclusión desde un orden institucional, curricular y metodológico a partir del virtuosismo tecnológico para garantizar el adecuado aprovechamiento de las estrategias y recursos digitales que se encuentran disponibles en la actualidad (Díaz \& Rodríguez, 2016).

La enseñanza desde un enfoque inclusivo, involucra un cambio hacia un nuevo paradigma educativo sustentado a partir de la equidad como elemento fundamental para fomentar la integración del estudiante sin distinción de condición (Navarro, 2015), siendo necesario para tal propósito, establecer una visión orientada a distinguir a cada uno de los estudiantes como individuos en capacidad de desenvolverse asertivamente en entornos de aprendizaje, de esta forma el rol del docente radica en guiar al estudiante en educación infantil haciendo énfasis en promover el descubrimiento de sus potencialidades (Ure, 2017).

En este sentido, la transición hacia una educación inclusiva requiere un cambio del modelo tradicional de enseñanza, en todas sus aspectos; consecuentemente al integrar estudiantes con diversidad de aprendizaje a los entornos de aprendizaje, es necesario que el docente pueda proporcionar un acompañamiento dirigido de acuerdo a su edad y el ritmo de sus capacidad individuales, así como también debe ser capaz de hacer uso adecuado de las herramientas tecnológicas para atender de forma adecuada las necesidades educativas de los estudiantes en etapa inicial. De esta forma se rompe el paradigma de uniformidad con el que se creía que todos los estudiantes debían aprender al mismo ritmo (Lagos \& Llancavil, 2016).

En la literatura moderna sobre la educación inicial de niños con discapacidad, el término inclusión comenzó a suplantar el concepto de integración utilizado anteriormente y en algunos casos, hasta cierto punto se presente como un enfoque contrapuesto, pretendiendo expresar con mayor precisión el cambio de comprensión de la realización de los derechos de las personas con diversidad de aprendizaje, y personas con otro tipo de discapacidad (Ministerio de Educación del Ecuador, 2018).

Estos escenarios deben direccionar a distinguir el modelo de integración por el de educación inclusiva, puesto que esto permite vislumbrar que los niños con diversidad de aprendizaje son individuos con capacidad de asumir retos en igualdad de condiciones, de modo que se propicie el aprendizaje en función de las competencias individuales que poseen y puedan ir desarrollando nuevas competencias y habilidades a lo largo de su etapa de formación inicial. Garantizar la equidad de condiciones en el entorno educativo, fomentará el aprendizaje y la adaptación de los niños con diversidad de aprendizaje, permitiéndoles reconocerse como personas generadoras de transformaciones individuales, en el entorno familiar y social en los que se desenvuelve (Peredo, 2016).

El problema que surgió durante la implementación de este cambio de paradigma radicó en el hecho de que la integración fue vista como la tarea de un individuo o un grupo de personas con necesidades educativas especiales, que debían adaptarse a las condiciones de las instituciones educativas regulares. Considerando que la inclusión tiene como objetivo cambiar el entorno de aprendizaje en las escuelas para que estas condiciones mismas se adapten a las necesidades de todas las categorías de estudiantes con y sin discapacidades. Esto da lugar al concepto de aprendizaje adaptado, que significa la adaptación de la escuela al alumno, y no al contrario, y está diseñado para acercar los enfoques de la pedagogía especial y los modelos de enseñanza inclusivos. En un ambiente educativo tanto el docente como los estudiantes con diversidad de aprendizaje, deben trabajar de forma cooperativa para alcanzar objetivos comunes (Molina, 2015).

En este sentido, si una visión estrecha se centra en un estudiante específico que tiene problemas en el proceso de aprendizaje, entonces una visión amplia considera los problemas del estudiante en el contexto del entorno de aprendizaje como un todo. Al mismo tiempo, siguiendo el modelo de educación inclusiva, la mayoría de los docentes comparten una visión que 
subyace en la idea que afirma la necesidad de tener en cuenta las necesidades educativas especiales y crear condiciones adecuadas para ello en las instituciones de educación infantil, de modo que un niño con diversidad de aprendizaje pueda estudiar junto con otros estudiantes que no presentan algún tipo de discapacidad (Grzona, 2014).

En el Ecuador, el Gobierno a través del Ministerio de Educación ha buscado promover la educación inclusiva desde el nivel de enseñanza inicial hasta niveles superiores, para ello han desarrollado programas mediante los cuales se ha restructurado el modelo educativo tradicional a fin de resaltar la incorporación, socialización, del estudiante con diversidad de aprendizaje, destacando además que esta pretende el desarrollo de escuelas inclusivas como modelo en donde se trabaja en la búsqueda de un equilibrar las habilidades y capacidades de los estudiantes (Duk \& Murillo, 2018).

Según los registros del Consejo Nacional para la Igualdad de Discapacidades del Ecuador, la diversidad de aprendizaje es la segunda de mayor representatividad porcentual, consecuentemente evidencia la necesidad de ser abordada en las instituciones de educación infantil y primaria desde un enfoque inclusivo con el adecuado aprovechamiento de la tecnología y las estrategias y materiales didácticos digitales que contribuyan a fomentar un aprendizaje creativo y significativo en los estudiantes en edades de 3 a 6 años con este tipo de discapacidad.

Sin embargo, para lograr dicho cometido es fundamental que el docente confíe vocacionalmente en sus habilidades formativas a fin de que pueda seleccionar las herramientas digitales adecuadas y que a su vez pueda ser asertivo en su rol, percepción que pueda ser transmitida a su vez a los estudiantes con el objeto de promover el desarrollo de sus capacidades y habilidades individuales (Sevilla-Santo, Martín-Pavón, \& Jenaro-Río, 2018).

El uso de las posibilidades tecnológicas en entornos inclusivos puede propiciar cambios en las posibilidades de aprendizaje completamente mejoradas por la tecnología. De esta manera se implementa el aprendizaje individual, inclusivo y descentralizado, orientado a las habilidades personales de los niños con diversidad de aprendizaje, y se brinda preparación para las condiciones de trabajo socialmente cambiadas. Actualmente existen diversos materiales didácticos disponibles en la web, como vídeos educativos que ayudan a los profesionales de la educación a integrar los medios digitales en entornos inclusivos. Sin embargo, considerando que los estudiantes con diversidad de aprendizaje deben ser percibidos como individuos con capacidades y ritmos de aprendizaje distintos entre sí, es necesario que el docente se haga la siguiente interrogante: ¿Qué estrategias didácticas se pueden usar para inventar historias creativas que favorezcan el aprendizaje? y ¿Qué materiales y herramientas son las más aptas para que todos los niños con distinto grado de diversidad de aprendizaje pueden realmente ponerlas en práctica, independientemente de si saben leer, escribir, o sostener el bolígrafo?

Según argumentan diversos autores, es posible aprender de forma individual e inclusiva con las posibilidades que ofrecen las tecnologías asociadas e implementar estrategias y materiales didácticos digitales que correspondan a las condiciones de trabajo socialmente cambiadas. Pero el uso de las tecnologías no es un éxito seguro, los resultados son tan buenos como los conceptos que se desarrollaron para ellos (Gómez, Laitón, Mejía, \& Sarmiento, 2017).

Es fundamental para la generación de instituciones educativas en etapa inicial inclusivas y la selección de estrategias y materiales didácticos digitales que se adapten a las necesidades de los estudiantes con discapacidad, conocer la población de niños de 3 a 6 años con diversidad de aprendizaje a nivel nacional y sus necesidades particulares de aprendizaje. En este caso, según el registro del Consejo Nacional para la Igualdad de Discapacidades del Ecuador actualizado al mes de septiembre del año 2020 un total de 2,065 niños con diversidad de aprendizaje en rango de edad señalado, de los cuales 12 pertenecen a la ciudad de Azogues (Consejo Nacional para la Igualdad de Discapacidades del Ecuador, 2020).

Los estudiantes con diversidad de aprendizaje o necesidades educativas especiales (NEE) en etapa inicial, además de un sistema educativo inclusivo apto para ofrecer las herramientas y materiales necesarios que faciliten su aprendizaje, requieren del soporte familiar como principal motivador, quienes también deben proporcionarles un entorno adecuado y de alta 
estima que le permita sentirse seguro y apoyado, trabajando de forma colaborativa con el docente para implementar estrategias y materiales didácticos digitales que favorezcan el aprendizaje de los niños (Calvo, Verdugo, \& Amor, 2016), consecuentemente es imperativo el despliegue de políticas educativas que propicien el trabajo conjunto entre la unidad educativa y la familia con la finalidad de fomentar la educación inclusiva de calidad desde un enfoque multidisciplinar.

No obstante, pese a que existen actualmente instituciones educativas especializadas en la enseñanza para niños con diversidad de aprendizaje, se ha identificado que existe un alto porcentaje de instituciones de educación infantil y primaria de enseñanza regular en la ciudad de Azoques que no son aptas para incorporar a estudiantes con diversidad de aprendizaje, lo cual acentúa un modelo educativo excluyente, considerando que según este enfoque los estudiantes con NEE son separados de los estudiantes que acuden a escuelas regulares.

Por lo tanto, a partir del nuevo paradigma el Gobierno ecuatoriano busca que las unidades educativas regulares sean adecuadas para integrar a todo tipo de estudiantes, para esto se requiere de diversos cambios y adaptaciones del sistema educativo, y el uso de metodologías, herramientas y materiales empleados en los procesos de enseñanza. Con base a lo descrito, el presente estudio tiene la finalidad de establecer la incidencia de las estrategias y materiales didácticos digitales y el aprendizaje en estudiantes de 3 a 6 años con diversidad de aprendizaje de la ciudad de Azogues, localizado en la provincia de Azuay del Ecuador.

\section{Metodología}

\subsection{Tipo de Investigación}

El estudio se desarrolló con base a un enfoque multi-método o modelo mixto que incluyó el paradigma cuantitativo y cualitativo, puesto que este enfoque empleado en investigaciones de carácter educativo puede proporcionar información complementaria y a profundidad sobre las variables observadas (Reis, Amorim y Melão, 2017). La investigación multi-método se basa en una estrategia de investigación metodológica que incluye más de un método de reunión de datos y/o más de un método de análisis de los datos; tales métodos pueden ser basado en técnicas cualitativas, técnicas cuantitativas o una mezcla de ambas (Seawright, 2016).

\subsection{Diseño de Investigación}

El diseño multi-método fue de tipo Anidado Concurrente de varios niveles (DIACNIV) recomendado por Hernández, Fernández y Baptista (2010). En este diseño, se estructura de tres fases o niveles, el primer nivel de tipo cualitativo, mediante análisis teóricos de diversos autores, luego en la segunda fase de tipo cuantitativa, se procede a la recolección de datos descriptivos estadísticos mediante un cuestionario, además, se aplicó un análisis descriptivo correlacional de Pearson basado en un estudio no experimental transeccional, considerando que se buscó analizar un escenario real, sin que exista injerencia por parte de los investigadores sobre las variables. En este caso, se buscó analizar el uso de estrategias y materiales didácticos digitales y el aprendizaje de los estudiantes con diversidad de aprendizaje de la ciudad de Azogues. Después de describir los datos numéricos, en la tercera fase de índole cualitativo, se procede a un análisis triangulado o contraste general de datos, que permitieron dar las conclusiones del presente estudio.

\subsection{Población y muestra}

En lo que respecta a la población de estudio, se trabajó con los estudiantes de 3 a 6 años quienes de acuerdo con los datos de registro del Consejo Nacional para la Igualdad de Discapacidades del Ecuador (2020), constan de un total de 12 individuos de los cuales 11 presentan diversidad de aprendizaje del 30\% al 49\%, mientras que el restante presenta un grado de 
discapacidad del 50\% al 74\%. Es importante mencionar que se trabajó con el total de la población, a través de la aplicación de un tipo de muestreo no probabilístico, puesto que se consideró únicamente a los estudiantes que residan dentro de la ciudad de Azogues (Mendoza, et al., 2021).

\subsection{Instrumentos de investigación}

Para llevar a cabo el levantamiento de la información, se trabajó con dos instrumentos. El primer instrumento fue la guía o ficha de observación digital. Debido a que no se realizan actividades presenciales en la Republica del Ecuador por motivos de prevención y cuidado de la Pandemia Covid-19, se tomaron medidas investigativas digitales, donde los investigadores, solicitaron al Consejo Nacional para la Igualdad de Discapacidades del Ecuador (2020), asistir de forma anexa no participativa en las actividades educativas implementadas por los docentes de la ciudad de Azogues. El primer formato o instrumento de recolección de datos incluyó criterios para evaluar el aprendizaje de los estudiantes con NEE (Tabla 1), para lo cual se realizó la adaptación de la estructura propuesta por el Ministerio de Educación del Ecuador (2019). Cada diseñó de instrumento se estructuro de acuerdo con cada una de las variables analizadas.

Tabla 1. Ficha de observación (Variable independiente: estrategias y materiales didácticos digitales).

Lugar de la evaluación:

Fecha de la evaluación:

Edad del estudiante evaluado:

Grado de diversidad de aprendizaje:

Criterios de valoración:

5: Muy bueno 4: Bueno

3: Regular

2: Bajo

1: Insuficiente

\begin{tabular}{ll}
\hline Criterio de evaluación & Valoración \\
\hline
\end{tabular}

1. Se aplican estrategias y materiales didácticos digitales ajustados a las necesidades y capacidades

de los estudiantes

2. Se trabaja con estrategias y materiales didácticos digitales que favorecen las interrelaciones entre

los estudiantes

3. Se trabaja con estrategias y materiales didácticos digitales que facilitan la comunicación de los estudiantes

4. Se trabaja con diferentes estrategias y materiales didácticos digitales en el aula

5. Se coordina con los familiares las acciones estratégicas y materiales didácticos digitales para atender a los estudiantes

6. Se respeta el nivel de desarrollo, estilos de aprendizaje, inteligencia y capacidades de cada estudiante

7. Se ofrece ayuda individualizada según las necesidades de cada estudiante

8. Se aplica estrategias y materiales didácticos digitales que favorecen el desarrollo lingüístico del estudiante

9. Se aplica estrategias y materiales didácticos digitales que favorecen la memorización y recuperación de información

10. Se aplica estrategias y materiales didácticos digitales basadas en trabajos grupales que fomentan el aprendizaje cooperativo

11. Se aplica estrategias y materiales didácticos digitales adaptativos para facilitar el desarrollo de habilidades útiles para resolver problemas de la vida diaria

12. Se aplica estrategias y materiales didácticos digitales que favorecen el aprendizaje funcional del estudiante

Fuente: Autores.

El segundo instrumento, tipo cuestionario incluyó preguntas con opciones de respuesta escalar tipo Likert con cinco alternativas (Tabla 2), considerando que en este caso se buscó evaluar las estrategias y materiales didácticos digitales aplicados por educadores en los procesos de enseñanza a este grupo de estudiantes (Ministerio de Educación, 2016). 
Tabla 2. Ficha de observación (Variable dependiente: nivel de aprendizaje).

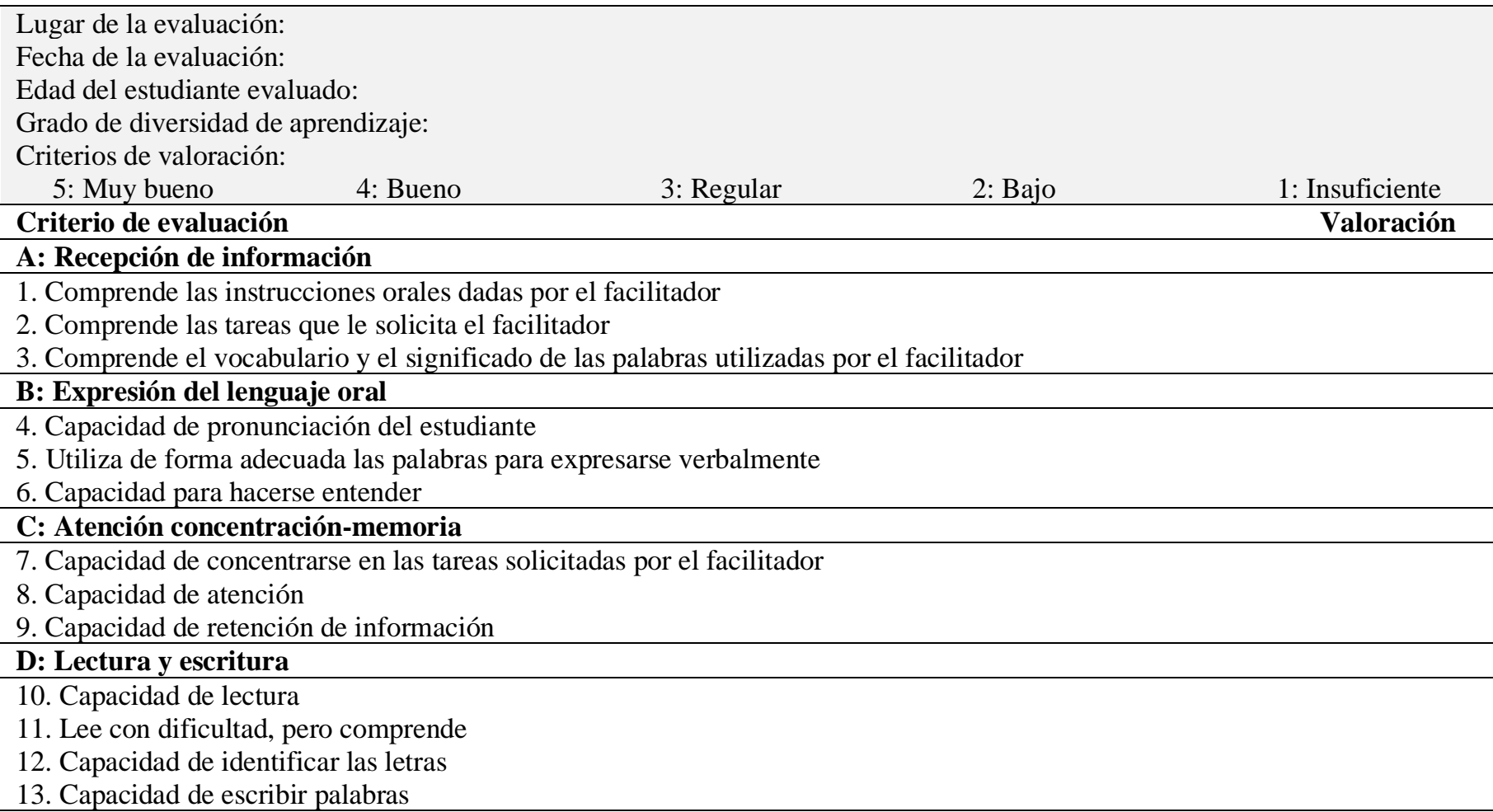
13. Capacidad de escribir palabras

\section{E. Matemáticas \\ 14. Capacidad para identificar los números cardinales}

Fuente: Autores.

Cabe mencionar que, en el caso de ambos instrumentos, se trabajó con criterios de evaluación sugeridos por el Ministerio de Educación de la Republica del Ecuador (2013), por lo tanto, constan de la respectiva validación requerida. Además, los dos instrumentos fueron validados por cinco expertos, también se aplicó el estudio de confiabilidad, mediante el coeficiente estadístico Alfa de Cronbach, donde se identificaron que para las estrategias didácticas un resultado de 0,870 y para aprendizaje de 0,890 es un valor confiable para su aplicación (Tabla 3). En este caso, para realizar el análisis e interpretación de los resultados, se tomó como referente los rangos de coeficiente que se presentan a continuación:

Tabla 3. Interpretación de coeficiente de Alfa de Cronbach.

\begin{tabular}{cc}
\hline Correlaciones positivas & Correlaciones negativas \\
\hline Entre 0.7 y 0.8 (Nivel aceptable) & Debajo de 0.5 (Nivel no aceptable) \\
Entre 0.8 y 0.9 (Nivel bueno) & Entre 0.5 y 0.6 (Nivel pobre) \\
Superior a 0.9 (Nivel excelente) & Entre 0.6 y 0.7 (Nivel débil) \\
\hline
\end{tabular}

Fuente: Mendoza et al., (2018).

\subsection{Técnica de análisis de resultados}

La información fue recolectada tomando como referencia las variables de estudio, tabulándose los datos a través del programa estadístico IBM SPSS Statistics versión 24, en la cual se determinó la correlación de Pearson. Patten y Newhart, (2017) resaltan que el coeficiente de correlación producto-momento de Pearson (o coeficiente de correlación de Pearson, para abreviar) es una medida de la fuerza de una asociación lineal entre dos variables y se denota por r. Básicamente, una correlación producto-momento de Pearson intenta trazar una línea de mejor ajuste a través de los datos de dos variables, y el coeficiente de correlación de Pearson r, indica qué tan lejos están todos estos puntos de datos de esta línea de mejor ajuste (es 
decir, cuánto bien, los puntos de datos se ajustan a este nuevo modelo / línea de mejor ajuste).

Particularmente las fases investigativas, consistieron en:

1. Observar el contexto de la investigación.

2. Desarrollar un análisis teórico documental (fase cualitativa).

3. Crear y validar los instrumentos de medición (fase cuantitativa).

4. Aplicar los instrumentos de medición para la obtención de información.

5. Procesar los datos estadísticos mediante la tabulación.

6. Triangular, preparar y presentar el informe escrito (Fase cualitativa).

Los datos obtenidos analizados en la tercera fase cualitativa fueron analizados mediante la técnica de triangulación o contrastación donde los resultados de los conjuntos de datos se analizan de forma grupal y comparativa. La visión alternativa es que la triangulación revela diferentes dimensiones de un fenómeno de investigación (Vogl, Schmidt \& Zartler, 2019).

\section{Resultados y Discusión}

Después de aplicarse los instrumentos de recolección de datos numéricos, se aplicó el análisis estadístico de correlación de Pearson evaluándose la variable independiente (VI: Estrategias y materiales didácticos digitales) con la variable dependiente (VD: Nivel de aprendizaje) (ver tabla 4).

Tabla 4. Correlación de las estrategias didácticas con el nivel de aprendizaje.

\begin{tabular}{cccc}
\hline & & VI & VD \\
\hline \multirow{2}{*}{ VI } & Correlación de Pearson & 1 & $0.639^{* *}$ \\
& Sig. (bilateral) & & 0.001 \\
& N & 12 & 12 \\
VD & Correlación de Pearson & $0.639^{*}$ & 1 \\
& Sig. (bilateral) & 0.025 & 12 \\
\hline
\end{tabular}

**. La correlación es significativa en el nivel 0.05 (bilateral)

Fuente: Autores.

Deforma descriptiva en el método de correlación de Pearson, los valores se ubican entre - 1 y 1 , donde 0 es sin correlación, 1 es correlación positiva total y -1 es correlación negativa total. Esto se interpreta de la siguiente manera: un valor de correlación de 0,639 entre dos variables indica que existe una relación significativa y positiva entre las dos. Una correlación positiva significa que, si la variable VI aumenta, entonces VD también aumentará, mientras que si el valor de la correlación es negativo, entonces si VI aumenta, VD disminuye (Nettleton, 2014). Según los resultados, en la Tabla 4 la variable independiente presenta una correlación alta de 0,639 con la variable dependiente (Nivel de aprendizaje), con una significancia bilateral de 0.05; por lo cual, se considera que es necesario que se sigan aplicando estas y otras estrategias innovadoras para mejorar el nivel de aprendizaje de los niños de 3 a 6 años con diversidad de aprendizaje de la ciudad de Azogues.

Las estrategias y materiales didácticos digitales van de la mano con los estilos de aprendizaje para aumentar los niveles de aprendizaje (Dörnyei, 2005). Los estilos de aprendizaje de los estudiantes se dividen en tres categorías: Estudiantes visuales, Estudiantes auditivos y Estudiantes kinestésicos. Estos estilos de aprendizaje se encuentran dentro del modelo VARK de aprendizaje estudiantil del teórico Dörnyei, (2005). VARK es un acrónimo que se refiere a los cuatro tipos de estilos de aprendizaje: visual, auditivo, preferencia de lectura / escritura y kinestésico. El modelo VARK reconoce que los estudiantes 
tienen diferentes enfoques sobre cómo procesan la información, lo que se conoce como "modos de aprendizaje preferidos".

En base a los resultados los estudiantes prefieren el modelo de enseñanza digital, como también, los recursos didácticos digitales, ya que de esta manera se sienten enlazados con el docente. Es decir, un estudiante post-moderno de la era de la conectividad, se siente a gusto con un docente que aplica recursos de su era digital. Betcy, Berry y Leah (2019) expresan que los modos de aprendizaje preferidos de los estudiantes:

- Tienen una influencia significativa en su comportamiento y aprendizaje.

- Deben combinarse con las estrategias de aprendizaje adecuadas.

- La información a la que se accede a través del uso de los estudiantes de sus preferencias de modalidad muestra un aumento en sus niveles de comprensión, motivación y metacognición.

Partiendo del estudio de referencia efectuado por Alvarado, (2016), el análisis previo de las estrategias aplicadas para estudiantes en educación infantil que presentan discapacidades de tipo intelectual permite identificar a su vez, las debilidades, requerimientos, necesidades, así como las fortalezas y competencias que cada niño presenta y con esto poder mitigarlas o potencializarlas. Entre las actividades didácticas se identifican las estrategias de ensayo, que consisten básicamente en hacer que los estudiantes sean expuestos repetidamente a un estímulo y que lo repitan constantemente para mejorar su comprensión sobre este, por ejemplo, el desarrollo del habla, pronunciación. Otro tipo de estrategias didácticas están basadas en la organización de información, que vaya desde lo más sencillo lograr su comprensión, y pasar posteriormente a lo más complejo, incentivando a la memorización y recuperación de la información. A su vez, se identifican las estrategias de talleres ocupacionales y de aprendizaje cooperativo, los cuales hacen alusión a actividades con fines terapéuticos para restaurar ciertas funciones comportamentales de los estudiantes en educación infantil, y para lograr que aprendan de manera conjuntan contenidos sobre ciencia, matemáticas, arte, sociales, entre otros.

En cuanto a los materiales didácticos digitales, según exponen Koppel, Suchodolki, y Zappalá, (2015), el entorno digital favorece significativamente a los alumnos que incluso han presentado falencias repetitivas en las clases convencionales, donde incluso pueden mejorar y adquirir nuevas habilidades y competencias: Los materiales didácticos digitales como una tablet, netbook o una computadora para que desarrollen actividades de dibujos, grabar sonidos, armar rompecabezas digitales, entre otras tereas, son las propicias para contribuir en el aprendizaje de los niños en educación infantil.

Por su parte Cegarra y García, (2015), acotaron que las estrategias y materiales didácticos digitales pueden estar más relacionadas con generar experimentación directa, la reflexión de los contenidos empleados, así como inculcando el aprendizaje por descubrimiento, esto mediante el uso de recursos de información y recursos multimedia para fortalecer la lingüística, memorización, habilidades de resolución de problemas, concentración, cálculos, entre otros. El desarrollo de las estrategias y materiales didácticos digitales, deben estar direccionadas para promover tanto el desarrollo, educación, bienestar y la reinserción de la persona a la sociedad y una plaza de trabajo (Peredo, 2016).

Dentro de las entidades especializadas a otorgar acompañamiento e incentivar el desarrollo y aprendizaje de estudiantes con discapacidades intelectuales, se considera entre los recursos más significativos a lograr, los docentes como a los propios alumnos, dado a que se busca generar la inclusión e inserción de estos últimos, es fundamental que la práctica docente sea la adecuada al manejar y establecer estrategias didácticas en los procesos de enseñanza con lo que se logrará niveles de aprendizajes que vayan de lo regular a lo excelente (Grande-Gonzáles, 2015). Es importante mencionar que dentro de los establecimientos educativos se logren constantemente evaluar los procesos, recursos, metodologías, estrategias y materiales, esto con el objetivo de corroborar si son las más idóneas de acuerdo a los grados de diversidad de aprendizaje que presentan los estudiantes en educación infantil, y por lo tanto, es necesario que a nivel público, el gobierno o los ministerios 
pertinentes, den seguimiento de lo que se imparte y aplica en estas instituciones para asegurar sobre todo, que sea una formación funcional (Villanueva, 2016).

Cabe acotar que las estrategias y materiales didácticos digitales presentados durante los participantes del estudio, se percibió un amplio abanico de opciones a nivel educativo y formativo, sin embargo, la aplicación de estos para mejorar el nivel de aprendizaje de los estudiantes en educación infantil los cuales dependerán de la metodología y forma que cada institución y facilitador aplique, así como será necesario un amplio nivel de preparación del profesional, dado a que requiere de compromiso, paciencia y constante autoaprendizaje debido a la complejidad que gira alrededor de la enseñanza-aprendizaje de los niños con discapacidades intelectuales.

\section{Conclusión}

Con base a lo analizado en los resultados en contraste con la revisión teórica, es posible determinar que las estrategias didácticas con relación al fortalecimiento del desarrollo lingüístico, memorización y recuperación de información, así como incentivar el trabajo en grupos, fortalecer las habilidades de aprendizaje cooperativo, resolución de problemas y fomentar el aprendizaje funcional, son estrategias que requieren un abordaje mucho más amplio. La estructura de la interacción en el aula dentro de estos grupos de estudiantes diagnosticados parece tradicional, es decir no se percibió una atención educativa de tipo racional o discriminativa.

Desde una perspectiva participativa y analítica, las actividades a distancia son problemáticas. Los estudiantes juzgan que necesitan un apoyo especial para su acto de aprendizaje en entornos de instrucción donde el docente domina claramente el discurso. Incluso cuando se anima a los estudiantes a discutir o ampliar temas, esto rara vez tiene éxito. La estrategia de enseñanza parece contribuir muy poco al diálogo con los estudiantes o entre los propios estudiantes, o a la construcción de algún tipo de conocimiento común en el aula. Es obvio que el docente promulga una tradición institucional de dar una lección sobre un tema determinado, lo que implica que el papel del estudiante es escuchar y proporcionar respuestas específicas de una palabra. Las contribuciones de los estudiantes son generalmente mínimas. Desde una perspectiva sociocultural, se enfatiza que el aprendizaje es una propiedad emergente de la interacción social, se puede concluir que hay poca evidencia de que los niños se vuelvan más activos o asuman un rol más participativo, habilidades necesarias para una ciudadanía activa en la sociedad actual.

De igual manera, los materiales didácticos digitales más frecuentes son las netbooks, computadoras o tabletas aplicadas para fortalecer las competencias creativas, de memorización, desarrollo lingüístico, entre otros. Estos recursos son los más propicios para lograr que los estudiantes en la etapa de educación infantil con diversidad de aprendizaje tengan un nivel de aprendizaje adecuado y eficiente. Con base a los cambios del entorno, para los docentes el estar preparados para el surgimiento de nuevas estrategias, o incluso crearlas por sí mismos, representará mayores oportunidades para este grupo vulnerable de personas en la búsqueda de erradicar la discriminación e incentivar su inserción a futuro en la sociedad.

\subsection{Recomendaciones}

Como recomendación se debe considerar que las prácticas educativas con estudiantes que evolucionan en estos entornos educativos se caracterizan principalmente por formas de enseñanza altamente individualizadas; la mayoría de las veces se puede percibir un docente instruyendo a un estudiante o dos. Incluso el aula bien sea virtual o presencial como escenario educativo, debe estar diseñada de una manera que se arroje evidencia del dominio de este tipo de instrucción o educación especial.

También se recomienda aplicar en futuras investigaciones la identificación analítica del docente, con respecto a la 
clasificación de estudiantes visuales, auditivos, de lectura / escritura, kinestésicos, aprendices y alinear los planes de estudios general, con estos estilos de aprendizaje resultará beneficioso para todos los estudiantes y así mejorar las cualidades cognitivas. Tener en cuenta, que a veces puede generar una combinación de todos los estilos de aprendizaje, las tres modalidades sensoriales pueden ser la mejor opción. Permitir que los estudiantes accedan a la información en términos con los que se sientan cómodos aumentará su confianza académica.

\section{Agradecimientos}

Agradecemos en primera instancia a las autoridades, docentes y estudiantes que se prestaron para efectuar la investigación en la ciudad de Azogues, Ecuador; dado a que su guía constante y apoyo con el que nos estimularon en el desarrollo de la investigación, lograremos muchas metas como parte del proceso educativo que continua mientras nos desenvolvamos dentro de esta área que demanda compromiso y autoaprendizaje.

\section{Referencias}

Abdul, J., \& Mohammed, M. (2019). Managing diversity: academic's perspective on culture and teaching. Race Ethnicity and Education, 22(5), 569-588. https://doi.org/10.1080/13613324.2017.1395325

Alvarado, M. (2016). Estrategias de enseñanza del docente para niños con capacidades diferentes. Ciudad de Guatemala: Universidad Rafael Landívar.

Bautista, M., \& Clavijo, R. (2020). La educación inclusiva. Análisis y reflexiones en la educación superior ecuatoriana. Alteridad Revista de Educación, 15(1), $114-124$.

Betcy, J., Berry, M., \& Leah, A. (2019). Course Format and Student Learning Styles: A Comparison of Political Science Courses. American Journal of Distance Education, 33(4), 262-275. 10.1080/08923647.2019.1643697

Bravo, G., Vallejo, P., \& Zambrano, G. (2019). Modelos de planificación educativa y diversidad en aulas de clases: La Rioja.

Calvo, M. I., Verdugo, M. Á., \& Amor, A. (2016). La Participación Familiar es un Requisito Imprescindible para una Escuela Inclusiva. Revista latinoamericana de educación inclusiva, 10(1), 99-113.

Cegarra, A., \& García, V. (2015). Intervención educativa en el alumnado con discapacidad intelectual. Murcia: Equipo de Orientación Educativa y Psicopedagógica Murcia.

Consejo Nacional para la Igualdad de Discapacidades del Ecuador. (septiembre de 2020). Registro de niños de 0-6 años con discapacidad intelectual. Obtenido de Consejo Nacional para la Igualdad de Discapacidades del Ecuador: https://www.consejodiscapacidades.gob.ec/estadisticas-de-discapacidad/

Consejo Nacional para la Igualdad de Discapacidades del Ecuador. (junio de 2020). Tipos de. Obtenido de Consejo Nacional para la Igualdad de Discapacidades del Ecuador: https://www.consejodiscapacidades.gob.ec/estadisticas-de-discapacidad/

Cruz, H., \& Giraldo, V. (2019). El Aprendizaje Flip Learning centrado en el estudiante como generador de calidad educativa: National University de San Marcos.

Díaz, L., \& Rodríguez, L. (2016). Educación inclusiva y diversidad funcional: Conociendo realidades, transformando paradigmas y aportando elementos para la práctica. Zona Próxima, O(24), 43-60.

Dörnyei, Z., (2005). Learning Styles and Cognitive Styles: Routledge.

Duk, C., \& Murillo, J. (2018). El Mensaje de la Educación Inclusiva es Simple, pero su Puesta en Práctica es Compleja. Revista latinoamericana de educación inclusiva, 12(1), 11-13.

Figueroa, M., Piñeres, C., \& Velásquez, J. (2017). Estrategias de inclusión en contextos escolares. Perspectivas en Psicología, 13(1), 13-26.

George, D., \& Mallery, P. (1995). SPSS/PC Step by step: a simple guide and reference. Belmont: Wadsworth Publishing Company: https://dialnet.unirioja.es/descarga/articulo/2476777.pdf

Gómez, S., Laitón, E., Mejía, C., \& Sarmiento, E. (2017). Competencia de prácticas inclusivas: las TIC y la educación inclusiva en el desarrollo profesional docente. Sophia, 13(2), 82-95.

Grzona, M. A. (2014). La accesibilidad educativa en las aulas inclusivas. una mirada didáctica. Investigación y Postgrado, 29(2).

Hernández, R., Fernández, C. \& Baptista, M. (2010). Metodología de la Investigación. México: McGraw Hill.

Koppel, A., Suchodolki, M., \& Zappalá, D. (2015). Inclusión de tic en escuelas para alumnos con discapacidad intelectual. Buenos Aires: Ministerio de Educación de la Nación. 
Lagos, L., \& Llancavil, D. (2016). Importancia de la educación inclusiva para el trabajo con niños con talento académico. Perspectiva Educacional, Formación de profesores, 55(5), 168-183.

Mendoza, D., Cejas, M., Navarro, M., Flores, E. \& Castillo, K. (2021). La eficacia pedagógica en la cultura organizativa escolar y la comunidad profesional de aprendizaje. Uniciencia, 35(2), e14490. http://dx.doi.org/10.15359/ru.35-2.11

Mendoza, D., La Madriz, J., López, M., \& Ramón, V. (2018). Research Competencies of Higher-Education Teaching Staff Based on Emotional Intelligence. Mediterranean Journal Of Social Sciences, 9 (5), 41. 10.2478/mjss-2018-0137

Ministerio de Educación. (2013). Educación especializada: Ministerio de Educación.

Ministerio de Educación. (2016). Instructivo para la evaluación y promoción de estudiantes con necesidades educativas especiales. https://educarecuador.gob.ec/anexos/ayuda/sasre/instructivo_de_evaluacion_de_estudiantes_con_nee.pdf

Ministerio de Educación. (2019). Adaptaciones curriculares para la educación especial e inclusiva. https://educacion.gob.ec/wpcontent/uploads/downloads/2019/05/Guia-de-adaptaciones-curriculares-para-educacion-inclusiva.pdf

Ministerio de Educación del Ecuador. (2018). Modelo nacional de gestión y atención: https://url2.cl/5VClu

Molina, Y. (2015). Necesidades educativas especiales, elementos para una propuesta de inclusión educativa a través de la investigación acción participativa. El caso de la Escuela México. Estudios pedagógicos, 41(especial), 147-167. https://dx.doi.org/10.4067/S0718-07052015000300010.

Navarro, M. J. (2015). Análisis de la inclusión educativa desde la perspectiva del profesorado de educación infantil, primaria, secundaria y bachillerato en el contexto educativo español. Investigación y Postgrado, 30(1), 33-55. http://ve.scielo.org/scielo.php?script=sci_arttext\&pid=S1316$00872015000100003 \& \operatorname{lng}=$ es\&tlng=es.

Nettleton, D. (2014). Commercial Data Mining: Morgan Kaufmann.

Patten, M. \& Newhart, M. (2017). The Pearson Correlation Coefficient (r): Routledge.

Peredo, R. (2016). Comprendiendo la discapacidad intelectual: datos, criterios y reflexiones. Revista de Investigación psicológica, (15), 101-122. http://www.scielo.org.bo/scielo.php?script=sci_arttext\&pid=S2223-30322016000100007\&lng=es\&tlng=es.

Reis, J., Amorim, M. \& Melão, N. (2017). Brea9 king barriers with qualitative multi-method research for engineering studies: Pros, cons and issues. Proelium $7(12), 275-292$

Romanelli, F., Bird, E., \& Ryan, M. (2009). Learning Styles: A Review of Theory, Application, and Best Practices. Am J Pharm Educ, 73(1), 19513146. https://www.ncbi.nlm.nih.gov/pmc/articles/PMC2690881/

Roque, D. (2018). Desarrollo personal y manejo asertivo de emociones en estudiantes. Episteme Koinonia, 1(2), 61-82. https://dialnet.unirioja.es/servlet/articulo?codigo $=7276125$

Seawright, J. (2016). Better Multimethod Design: The Promise of Integrative Multimethod Research. Security Studies, 25(1), 42-49. $10.1080 / 09636412.2016 .1134187$

Sevilla-Santo, D., Martín-Pavón, M. J., \& Jenaro-Río, C. (2018). Actitud del docente hacia la educación inclusiva y hacia los estudiantes con necesidades educativas especiales. Innovación educativa, 18(78), 115-141.

Ure, M. (2017). De la alteridad a la hiperalteridad: la relación con el otro en la Sociedad Red. Sophia, Colección de Filosofía de la Educación, 22 (1), 193-212.

Villanueva, G. (2016). Estrategias pedagógicas para discapacidad: Universidad Santo Tomas.

Vogl, S., Schmidt, E., \& Zartler, U. (2019). Triangulating perspectives: ontology and epistemology in the analysis of qualitative multiple perspective interviews. International Journal of Social Research Methodology, 22(6), 611-624. 10.1080/13645579.2019.1630901 